\title{
Multiscale Mechanics of Chalk from X-ray Tomography Data
}

\author{
$\underline{\text { Dirk Müter }}^{1, *}$, Stefan Bruns ${ }^{1}$ and Henning O. Sørensen ${ }^{1}$ \\ ${ }^{1 .}$ University of Copenhagen, Nano-Science Center, Department of Chemistry, Copenhagen, Denmark \\ *mueter@nano.ku.dk
}

Chalk is a biogenic limestone found in many countries bordering the North Sea. It hosts both groundwater and hydrocarbon reserves, thereby playing an important economic factor for countries such as the UK, Norway and Denmark. It is formed from the shield-like remains (coccoliths) of ancient coccolithosphores, one celled algae. A large part of these micrometer sized coccoliths, depending on the conditions (pressure, temperature, etc.) under which the rock was formed, did never recrystallize. This leads to pore sizes in chalk that are on the nanometer and submicrometer scale. Traditionally, chalk has been studied using standard laboratory analysis of centimeter sized cylinders (core plugs) taken from larger cores drilled from explorations wells. This process is a very costly and basically treats the rock as a black box. Imaging of chalk using X-ray tomography provides us with a direct view of the rock microstructure and its pore network, which can help us to, not only learn more about the rock forming process but also to reduce exploration costs by deriving petrophysical parameters from the tomography data and upscaling them to the field scale.

To image the microstructure of this rock using X-ray tomography and resolve the small features found in the coccoliths, we have to use nanotomography [1]. However, higher resolution comes at the cost of a smaller field of view which means a large amount of samples would be needed to retrieve statistically relevant information and additionally larger scale features such as microfractures may be lost. To overcome this limitation, we present a method to combine synchrotron based nanoCT with laboratory based microCT and demonstrate how to derive elastic mechanical properties using this multiscale modelling approach.

We took small samples $(\sim 100 \mu \mathrm{m})$ from four core plugs of Upper Maastrichtian chalk from the North Sea Basin and recorded ultra-high resolution nanotomography data at beamline BL47XU at SPring-8, Japan (Fig. 1a, inset) with a voxel size of $\sim 40 \mathrm{~nm}$. Furthermore, microCT data (Fig. 1a) on millimeter sized samples from the same core plugs were collected using an Xradia Versa 420 instrument at the Technical University of Denmark with a voxel size of about $1 \mu \mathrm{m}$. Figure 1 shows that the pore system is well resolved in the nanoCT data whereas in the microCT data only the largest pores can be seen and the smaller pores are blurred. However, larger features such as dense regions can be easily recognized in the microCT data. We first enhance the image quality using an iterative non-local means algorithm [3] and then segment the data using the watershed algorithm. From the segmented nanoCT data, we crop (6 $\mu \mathrm{m})^{3}$ sized subvolumes and mesh the data in the finite element software package FEBio. A uniaxial tensile testing environment is set up by prescribing a fixed boundary condition to one side of the mesh and a tensile load to the opposite side. From the deformed state simulated with the finite element method, we were able to calculate the strain along the loading direction, which in combination with the known applied load yields the effective Young's modulus. Repeating this procedure for a large number of subvolumes and samples taken from the same core plug we can produce a characteristic porosityelasticity relationship from nanoscale data on micrometer sized material volumes [2].

In order to link these nanoscale properties to the microscale, we first estimate the porosity of each voxel 
based on the greyscale value using dense regions and hollow fossils as markers for 0\% and 100\% porosity and scaling the voxel values between these. We assign material properties to each voxel in the microCT by using the nanoscale porosity-elasticity relationship and set up a similar tensile testing environment for subvolumes $(80 \mu \mathrm{m})^{3}$ of the microCT data (Fig. 1b), where we employed a uniform hexahedral mesh to have each voxel correspond to one hexahedral element. With this setup, we were able to derive the effective Young's modulus on the microscale while explicitly using the results from the nanoscale. Thus, we have effectively created a multiscale model that combines the high resolution of synchrotron based nanoCT with the larger field of view of laboratory microCT. Our preliminary results depicted in Figure 1c show that the nanoscale porosity-elasticity relationship is quite accurately reproduced on the microscale for some samples but not for all. The origin of this difference is subject to further studies [4].

[1] D Müter et al, Appl. Phys. Lett. 105 (2014) 043108

[2] D Müter et al, Nanoscale mechanical properties of porous materials from ptychographic X-ray imaging. In preparation

[3] S Bruns, SLS Stipp and HO Sørensen, Adv. Water Res. 105 (2017) 96-107

[4] The authors acknowledge funding from the Innovationfund Denmark and Maersk Olie og Gas A/S via the $\mathrm{P}^{3}$ project and the Danish Agency for Science, Technology and Innovation via Danscatt.
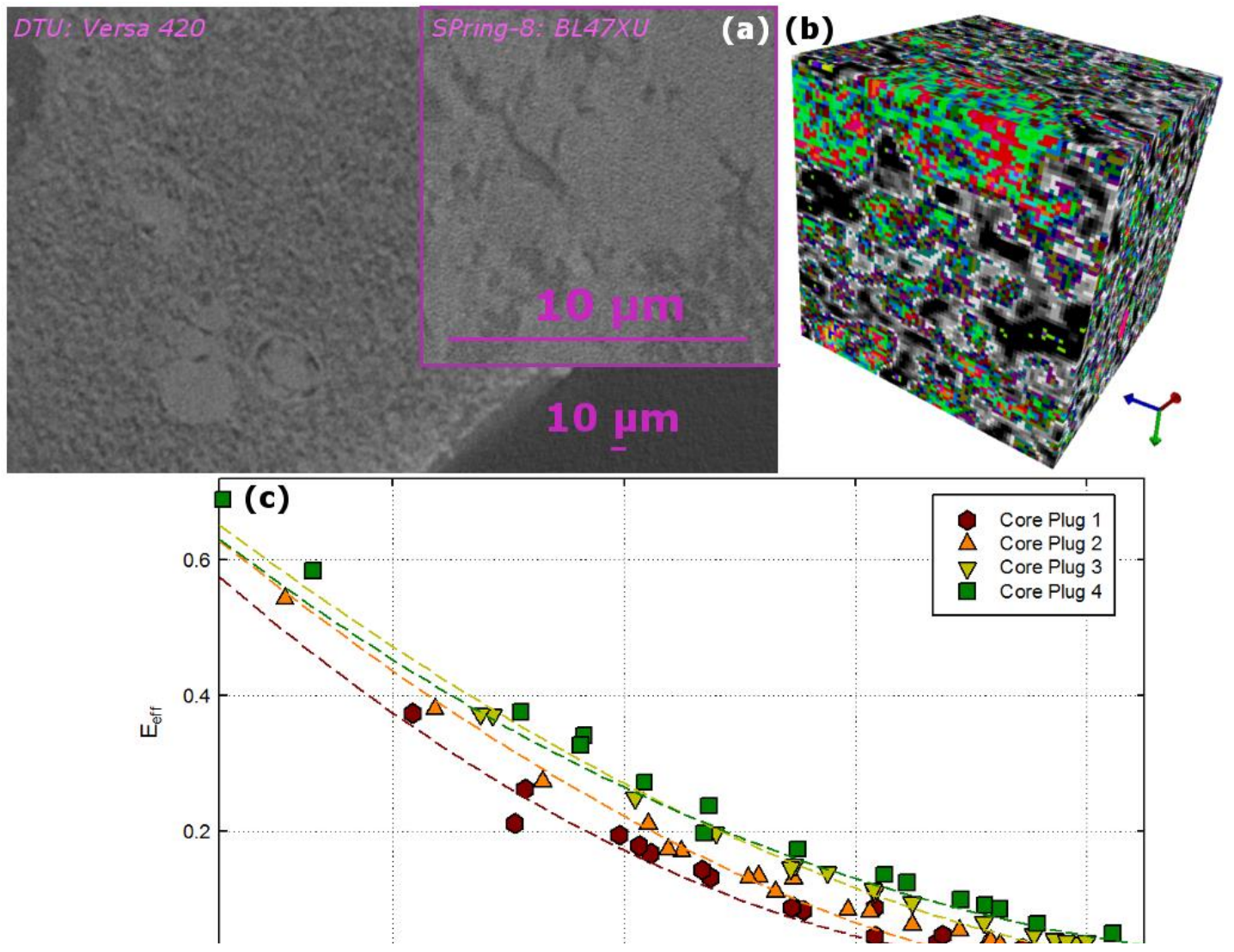

Figure. 1. (a) Slices of tomography data on chalk recorded on a laboratory microCT instrument at DTU and at beamline BL47XU, SPring-8, Japan (inset). (b) Subvolume of the microCT data (80 voxels edge length) imported and meshed in finite element software. The color code corresponds to local effective Young's modulus determined by the porosity-elasticity relationship derived from the nanoCT (synchrotron) data. (c) Effective Young's modulus derived from microCT data using the nanoscale elasticity-porosity relationship (dashed lines). 